\title{
Radiation Retinopathy-Clinical, Histopathological, Ultrastructural and Experimental Correlations
}

\author{
D. B. ARCHER, W. M. K. AMOAKU and T. A. GARDINER \\ Belfast
}

\begin{abstract}
Summary
Clinical, pathological and experimental studies of radiation retinopathy confirm that the primary vascular event is endothelial cell loss and capillary closure. Pericytes are less susceptible, but typically atrophy as the capillaries become nonfunctional. The immediate effects of radiation reflect interphase and early mitotic death of injured endothelial cells, whereas later changes may be attributed to delayed mitotic death of compromised endothelial cells as they attempt division in the ordinary course of repair and replacement. Capillary occlusion leads to the formation of dilated capillary collaterals which may remain serviceable and competent for years. Microaneurysms develop in acellular and poorly supported capillaries, predominantly on the arterial side of the circulation and adjacent to regions of poorly perfused retina. Alterations in haemodynamics produce large telangiectaticlike channels which, typically develop a thick collagenous adventitia and may become fenestrated. Limited capillary regeneration occurs, usually evident as recanalisation of arterioles or venules by new capillaries. Vitreo-retinal neovascularisation may occur where retinal ischaemia is widespread. Radiation produces an exaggerated vasculopathy in patients with diabetes mellitus, and five month streptozotocin-induced diabetic rats develop a severe ischaemic retinopathy with vitreoretinal neovascularisation when exposed to $1500 \mathrm{cGy}$ of radiation. Later photocoagulation is useful in containing or reversing microvascular incompetence and vasoproliferation in some patients with advanced radiation retinopathy.
\end{abstract}

The deleterious effects of ionising radiation on the retinal vasculature were first identified by Moore $^{1}$ in 1935, who described an ischaemic retinal vasculopathy in patients with retinoblastoma treated by radium seeds. His reports were confirmed by Stallard, ${ }^{2}$ Hay$\mathrm{reh}^{3}$ and others who demonstrated that a wide spectrum of retinal vascular pathology could follow brachytherapy, including vascular occlusion, incompetence and proliferation, and profound alterations in the architecture of the affected microvasculature. The chor- oidal circulation was similarly compromised at the site of the applied radioactive plaque.

Teletherapy, usually in doses exceeding $2,500 \mathrm{cGy}$, produced similar retinal vascular changes, ${ }^{4,5,6}$ although the choroidal circulation appeared relatively unaffected. Merriam ${ }^{7}$ and more recently Elhers and $\mathrm{Kaae}^{8}$ have reviewed the literature, emphasising the relationship between the extent and severity of the retinopathy and the radiation dose, ${ }^{9}$ fractionation schedule, concomitant use of chemotherapy and coexistence of diabetes

From: Department of Ophthalmology, The Queen's University of Belfast.

Correspondence to: Professor D. B. Archer, Department of Ophthalmology, Eye \& Ear Clinic, Royal Victoria Hospital, Belfast BT12 6BA, Northern Ireland. 


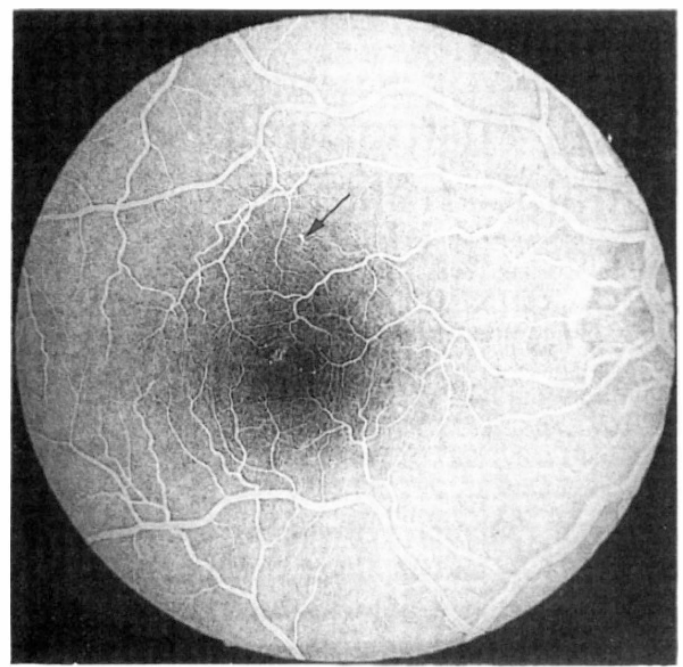

Fig. 1. Mid-venous phase angiogram of patient who received 5,250 cGy for a nasopharyngeal tumour. Irregular capillaries and a microaneurysm (arrow) indicate the presence of early radiation retinopathy.

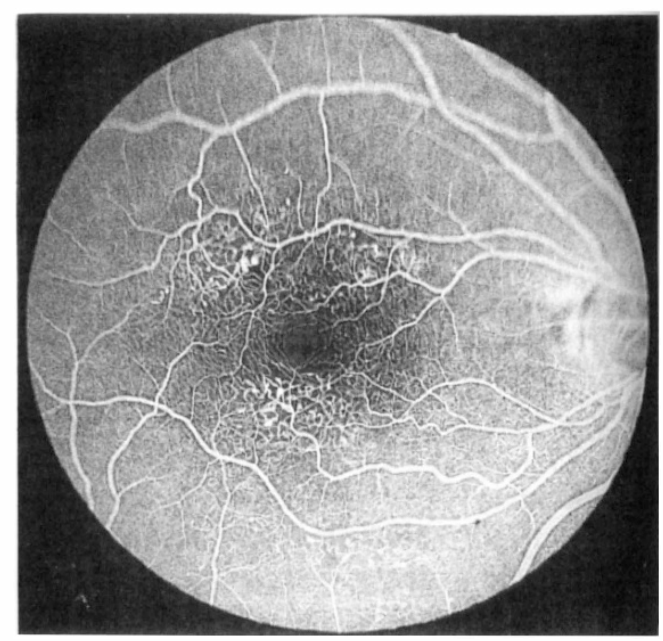

Fig. 2. Venous phase angiogram of patient, treated with 5,000 cGy of radiation for a nasopharyngeal tumour. The macular microvasculature is irregularly dilated and the site of numerous microaneurysms. Small areas of capillary non perfusion are present.

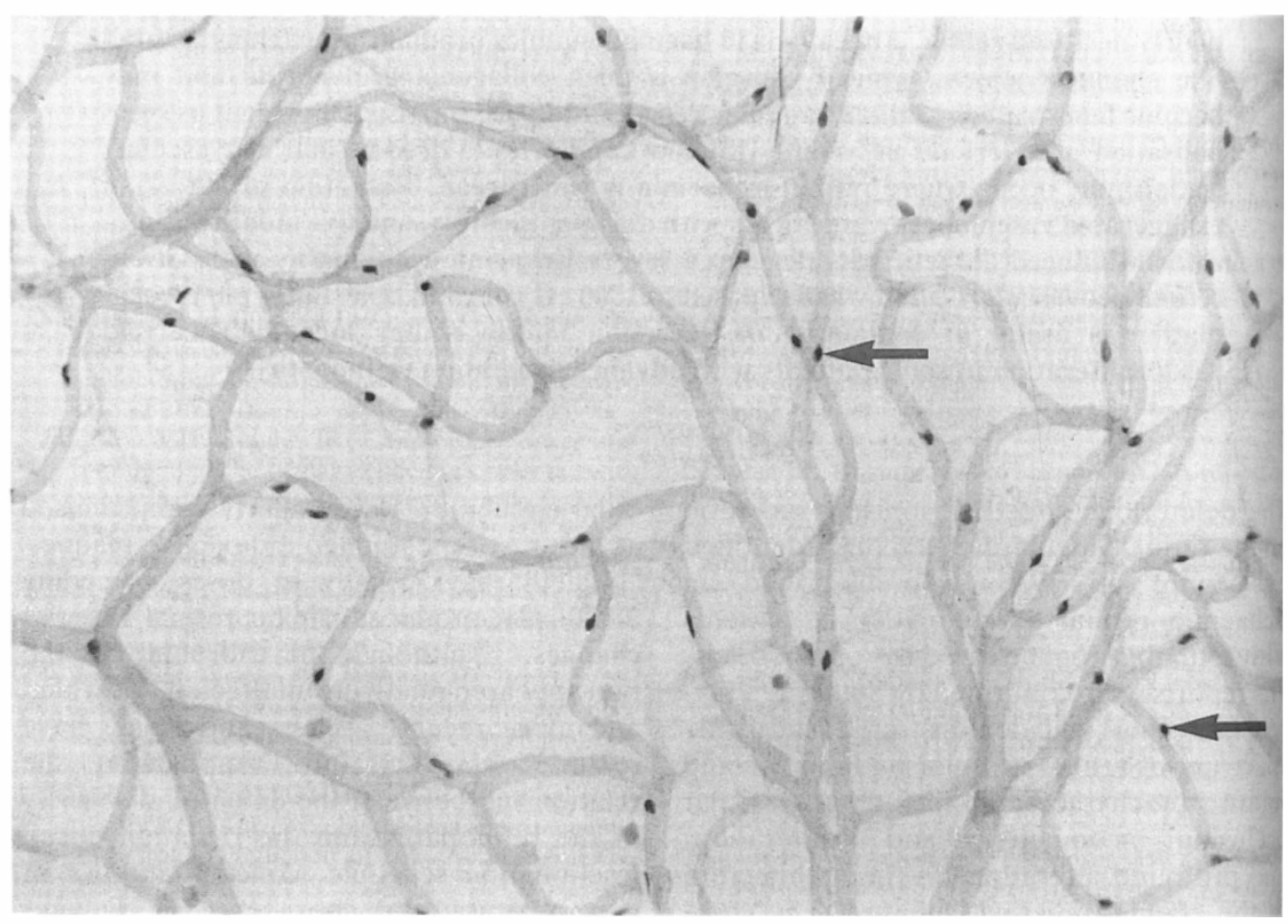

Fig. 3. Retina from a 59-year-oldnon-insulin dependent diabetic 18 months after 5,500 cGy of 8 MV photons. The patient was diagnosed diabetic one year prior to irradiation and showed no evidence of diabetic retinopathy. Trypsin digest preparation of the retinal vasculature shows extensive loss of endothelial cells in the retinal capillaries but relative sparing of the pericytes (arrows). Orig. Mag. $\times 250$. 
mellitus. ${ }^{10}$ The variable and often unpredictable latent period between radiotherapy and the development of clinically observable retinopathy has been highlighted by numerous authors. $^{6-13}$ Kinyoun et $a l,{ }^{14}$ and others ${ }^{15-19}$ have shown that laser photocoagulation, in certain circumstances, can contain or reverse vascular incompetence and neovascularisation with preservation or improvement in visual functions.

Despite the wealth of knowledge about established and end-stage radiation retinopathy, relatively little is known about the early retinal vascular changes and their evolution, and in particular the initial events in the retinal vascular cells that precipitate vascular incompetence, occlusion and proliferation. This paper reviews the vascular changes in radiation retinopathy, correlates the clinical findings with histopathological and ultrastructural alterations from post-irradiation, enucleated human eyes, and considers the response of the rat retinal vasculature to single doses of ionising radiation as a suitable model.

\section{Methods and Results}

Radiation Retinopathy-Clinical and Angiographic Features

Data was derived from the study of 18 patients in Northern Ireland who received cephalic radiation between 1972 and 1988. All patients received teletherapy from the $8 \mathrm{MV}$ linear accelerator (high energy photons), cobalt 60 machine (gamma rays), or X-rays from a 300 KV plant. All patients had a full ocular examination and documentation of visual functions. The detailed results of radiation dose, fractionation schedules and indications for radiotherapy are reported elsewhere. ${ }^{6,19,20}$ Fifteen patients ( 22 eyes) had fluorescein angiography. The earliest clinically observable changes were foci of dilated capillaries and microaneurysms occurring singly or in small clusters (Fig. 1). Fluorescein angiography highlighted local changes in vascular architecture, especially capillary fusion and irregularity and non-perfusion of some small channels in the immediate vicinity of the abnormally dilated vessels. The clinical and angiographic

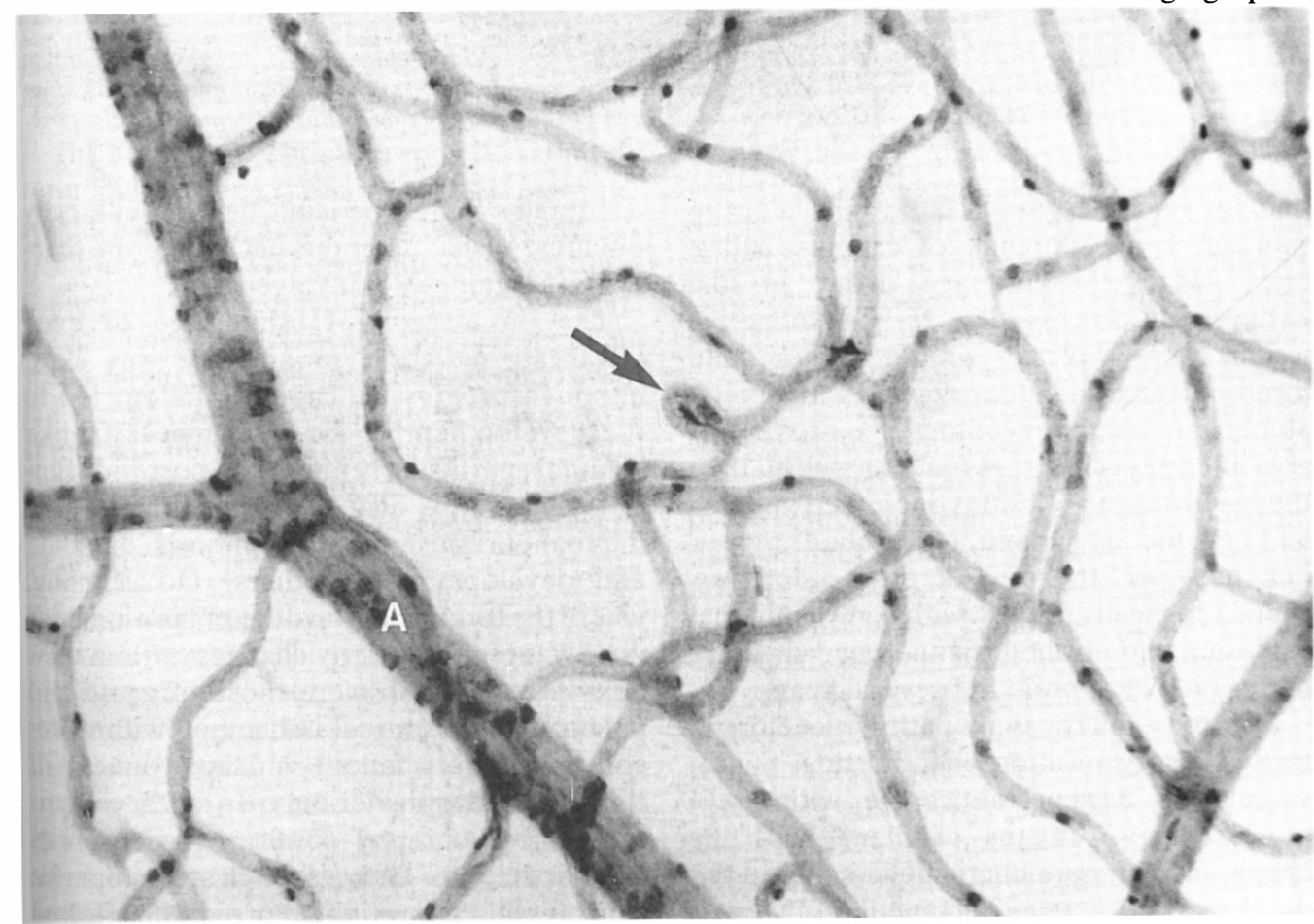

Fig. 4. Human retina from a 68-year-old woman six months after 4,500 cGy of $8 \mathrm{MV}$ photons. Trypsin digest preparation of the retinal vasculature shows an early microaneurysm (arrow) close to a retinal arteriole $(A)$. Orig. Mag. $\times 250$ 


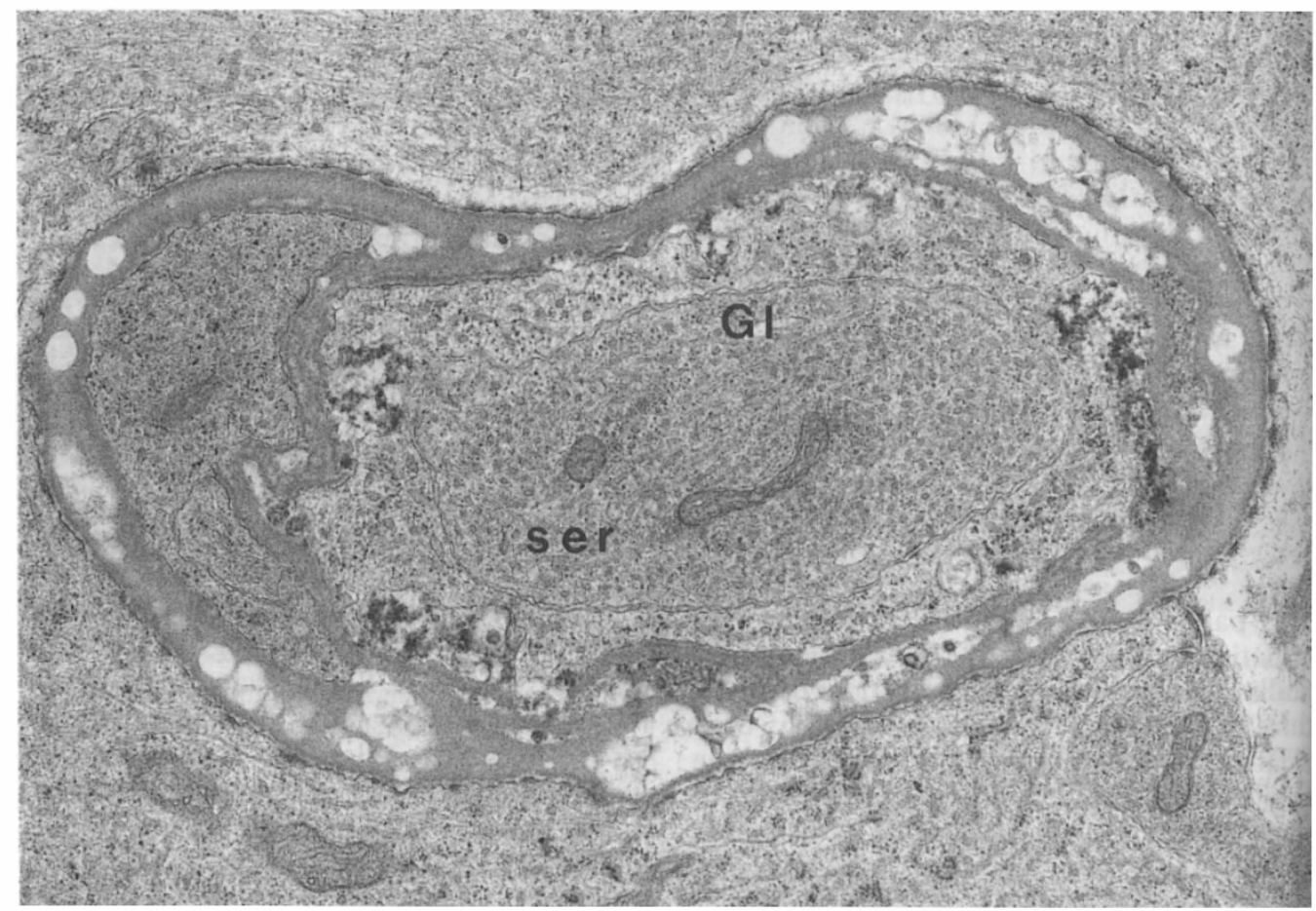

Fig. 5. Electron micrograph of retina from patient described in Fig. 3. A redundant tube of vascular basal lamina is filled with processes of hypertrophic glial cells $(\mathrm{Gl})$ containing extensive arrays of smooth endoplasmic reticulum (ser). The basal lamina shows the typical 'swiss cheese' vacuolation common in elderly human retinal vessels. Orig. Mag. $\times 9000$

findings suggested that focal capillary occlusion and the development of 'capillary collaterals' were the primary event in the pathological process. The early retinopathic lesions were typically subtle, minute, and localised and were not associated with detectable microvascular incompetence or measurable alterations in visual functions. With time the microvascular lesions increased in area and new foci developed in neighbouring retina; however, the rate of progression was characteristically slow, with only minimal extension of deformed channels and capillary occlusion over a period of several years.

As the microvasculopathy developed, further disorganisation of the retinal microvasculature became noticeable with additional collapse of the capillary bed, the appearance of more microaneurysms and the development of telangiectatic-like channels which straddled areas of poorly perfused retina (Fig. 2). Many of these abnormally dilated channels were incompetent to dye and eventually retinal oedema became clinically obvious. Visual functions declined if the macula was significantly involved.

The progression of established or even advanced radiation retinopathy was slow, although progressive capillary fallout led to the development of fresh telangiectatic-like channels particularly about the posterior fundus. There was also a gradual turnover of microaneurysms, with thrombosis of some and development of others. Occasionally, where the total radiation dose or fractionateddose was high, the early changes, after a variable latent period, were those of acute and extensive inner retinal ischaemia, with widespread capillary fallout and involvement of the precapillary arterioles. In such circumstances angiography confirmed widespread capillary closure and, although some reperfusion of ischaemic retina occurred, the affected retina remained non-functional and visual functions were predictably poor especially if the macula was implicated. 




Fig. 6. Electron micrograph of retina from patient described in Fig. 3 shows a new intraretinal vessel (lumen L) from the parafoveal region. The hypertrophic endothelial cells (E) show numerous fenestrations (arrows). Orig. Mag. $\times 5200$

The long-term complications of radiation retinopathy were the consequence of inner retinal ischaemia and vascular incompetence, i.e. intraretinal and preretinal neovascularisation, vitreous haemorrhage and retinal detachment or persistent macular oedema with cystoid degenerative changes and foveolar atrophy. Photocoagulation was used in three patients in this study to limit vasoproliferation and improve microvascular competence and was successful in either stabilising or improving visual functions.

\section{Radiation retinopathy-Histology and} Electron Microscopy

Clinico-pathological observations were drawn from seven eyes enucleated following therapeutic ocular radiation and evaluated by trypsin digest, routine histopathology and electron microscopy. ${ }^{20}$ Trypsin digest specimens in early vasculopathy demonstrated a preferential loss of vascular endothelial cells with relative sparing of the pericyte population(Fig. 3). Changes in capillary architecture were common, particularly outpouchings, fusiform annular dilatations and microaneurysms which predominated on the arterial side of the circulation (Fig. 4). More severely affected retina revealed acellular capillaries with the residual basement membrane tubes being typically fused, shrunken or collapsed.

Electron microscopy also identified a wide range of microvascular alterations, the principal feature again being a striking loss of vascular endothelial cells in affected vessels. The pericytes appeared more resistant to radiation damage and often survived within the walls of unlined vascular basement membranes. Severely damaged capillaries and arterioles exhibited defunct and acellular basement membranes, some of which were invaded by glial cells (Fig. 5) or recanalised by new intraretinal vessels. These new intraretinal vessels consisted of hypertrophic endothelial cells and pericytes and had elaborated their own basal lamina within that of the atrophic vessel. Some of the newly formed intraretinal capillaries were unusual in that they had fenestrated endothelial cells (Fig. 6).

Some larger calibre vessels which were dif- 


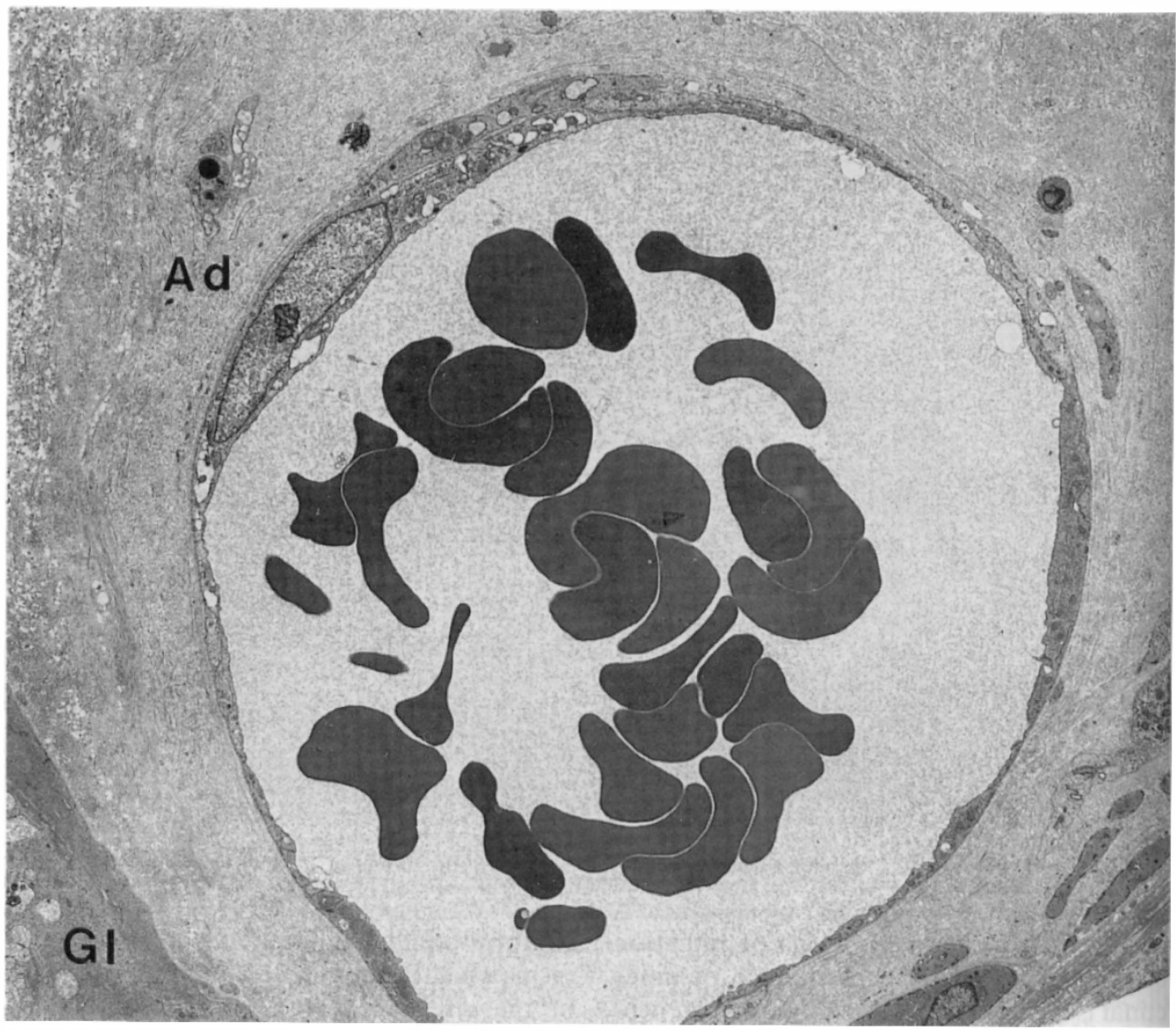

Fig. 7. Electron micrograph of retina from patient described in Fig. 3 shows a telangiectatic vessel from the paramacular region. The vessel has an attenuated endothelium which is separated from the surrounding glia (Gl) by a dense adventitia $(A d)$ containing large accumulations of fine collagen fibrils. Orig. Mag. $\times 6500$

ficult to define ultrastructurally as either arterioles or venules probably corresponded with the telangiectatic-like radicals noted particularly on fluorescein angiography and were characteristic of more advanced radiation retinopathy. These vessels were typically ensheathed by a dense adventitia of fine collagen fibrils and their endothelial cells were often fenestrated (Fig. 7). The retinal parenchyma in the region of such vessels was usually atrophic, containing haemorrhagic debris, hypertrophic glial cells and degenerate or swollen neurones. Macrophages, some of which had the features of 'foam cells' were present throughout the layers of the partially degenerate retina.

\section{Radiation Retinopathy-Experimental Vasculopathy}

Hooded male Lister rats were subjected to single doses of radiation between 200 and 2,000 cGy. The most pronounced changes were noted six months after exposure of the eye to $1500-2000$ cGy of KPV X-rays..$^{20}$ Trypsin digest specimens showed that the earliest changes in affected vessels were elongation of the endothelial cell nuclei and narrowing of the capillary lumen. Other vessels showed complete loss of endothelial cells and capillary collapse (Fig. 8), although pericytes often remained viable and persisted within the wall of the unlined basement membrane tube (Fig. 9). In severely affected retina only acellular 


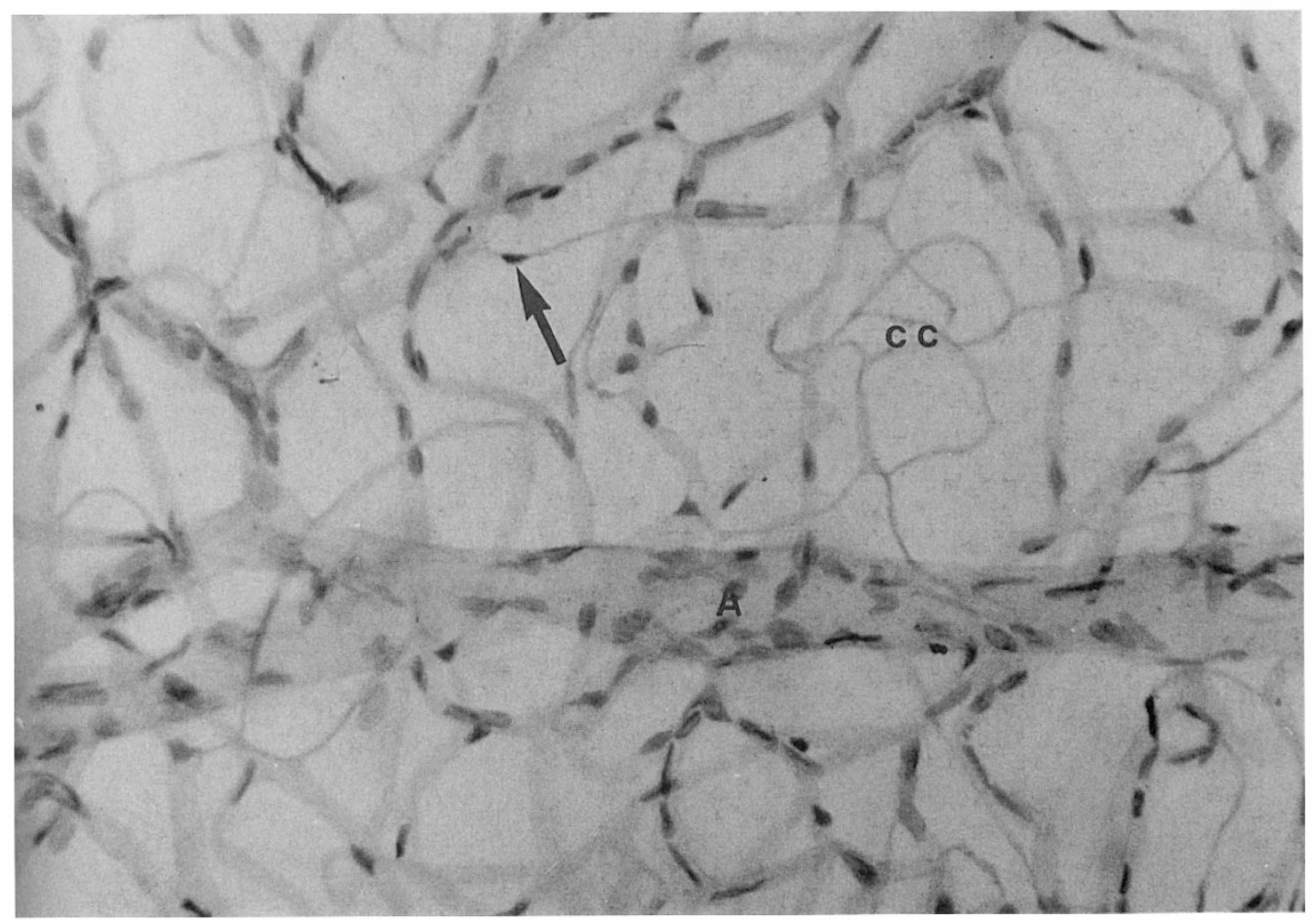

Fig. 8. Trypsin digest preparation of rat retinal vasculature six months after 1,500 cGy of $x$-radiation. Small confluent areas of capillary closure are present (cc), the acellular capillaries having collapsed to leave vascular strands. A capillary strand with a viable pericyte within its wall is arrowed. The retinal artery $(A)$ has lost the normally regular arrangement of smooth muscle cells. Orig. Mag. $\times 250$

vascular basement membranes remained and these were reduced to fine, strand-like structures. Microaneurysms were not present and no intraretinal or preretinal neovascularisation was identified in this study.

Changes in vascular cell turnover were evaluated by labelling S-phase cells with tritiated thymidine (Fig. 10) using an intraperitoneal osmotic mini-pump to deliver the isotope. Little change in vascular cell kinetics was noted in the short term, i.e. one to two weeks, following 1000-2000 cGy of radiation. However, at six months following irradiation, animals which had received doses of 1000 $1500 \mathrm{cGy}$, showed a five to ten fold increase in the number of microvascular cells engaged in DNA synthesis, measured over a 24 hour period. This striking increase in the labelling index of microvascular cells coincided with the post-irradiation loss of endothelial cells observed in trypsin digest and ultrastructural studies, and suggested that accelerated cell division was taking place in an attempt to replace endothelial cells lost due to radiationinduced mitotic death or delayed interphase death.

In view of the known susceptibility of the retinal vasculature in diabetic patients to ionising radiation, an experiment was conducted in which streptozotocin (STZ) induced diabetic Wistar rats had one eye irradiated with 1000-1500 cGy of $90 \mathrm{KVP}$ X-rays at three, five and 8.5 months after induction of diabetes. Animals given streptozotocin but remaining euglycaemic and normal rats served as controls. The most profound microvascular changes observed in trypsin digest specimens and electron microscopy occurred in the retinae of animals which had been diabetic for 5-8.5 months and then given 1000$1500 \mathrm{cGy}$ of radiation to the eye. The principal ultrastructural findings were endothelial cell degeneration and the presence of acellular and grossly thickening basal lamina tubes. Some capillaries displayed pericyte loss in the presence of healthy endothelial cells and vice 


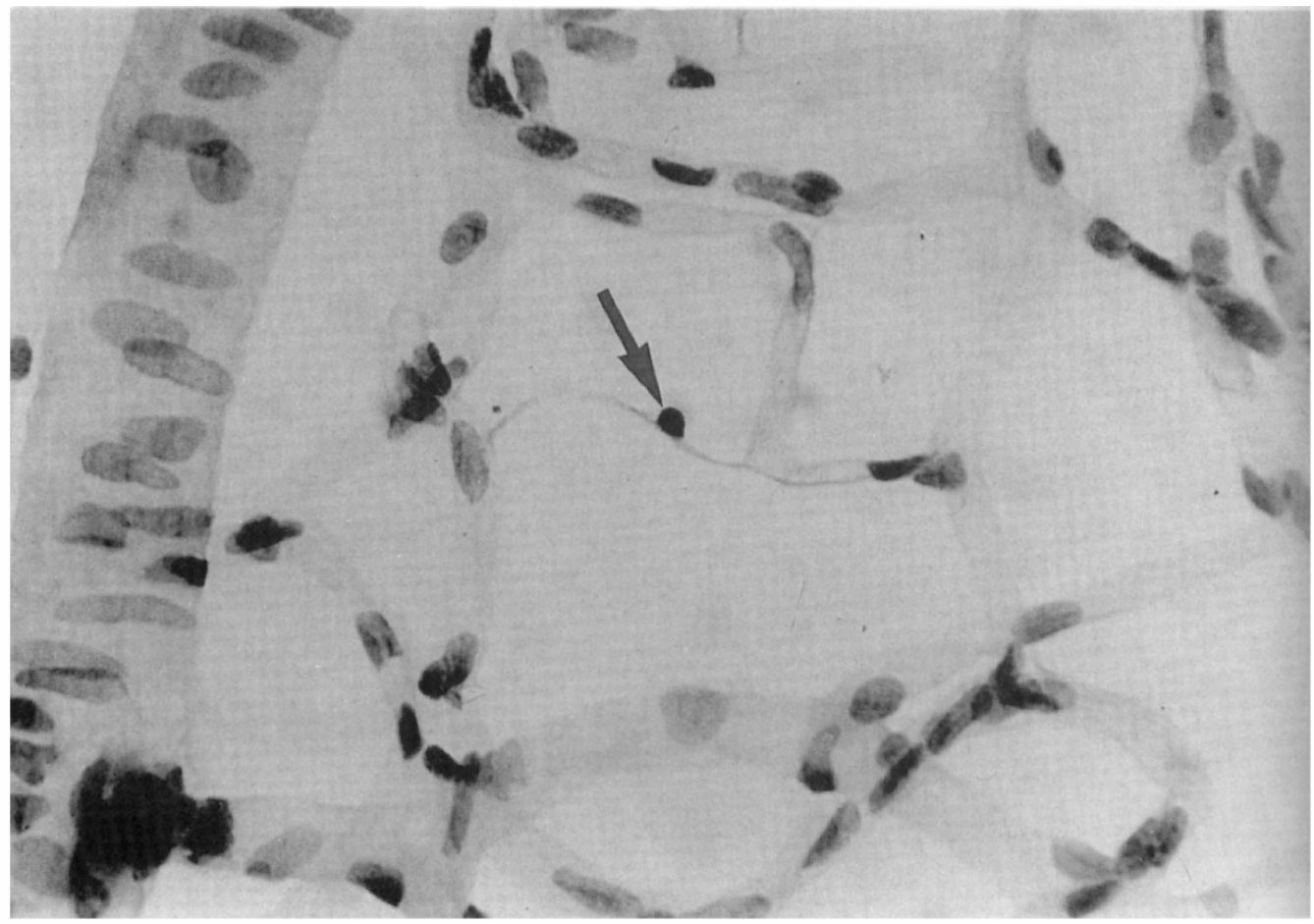

Fig. 9. Trypsin digest of rat retinal vasculature six months after 1,000 cGy of $x$-radiation. A stained pericyte nucleus (arrow) can be seen in the wall of a capillary strand. Orig. Mag. $\times 500$

versa. Some arterioles showed smooth muscle cell loss.

New intraretinal and preretinal vessels were also observed in two animals. These vessels proliferated within cystoid spaces in the partly degenerate and ischaemic inner retina which had numerous swollen and deformed axons reflecting impaired axoplasmic transport. These new vessels subsequently breached the internal limiting membrane to propagate on the surface of the retina and within the vitreous cavity (Fig. 11). The endothelial cells of the intravitreal new vessels were fenestrated and had a fine flocculant basal lamina. Large pericytes with rich complements of cytoplasmic organelles typically surrounded the intravitreal vessels (Fig. 12) but were less evident at the advancing front of the neovascular membrane.

\section{Discussion}

The susceptibility of the retina and its vasculature to high doses of ionising radiation delivered by either brachytherapy or tele- therapy has been known for some time and the various responses of the retinal and choroidal circulations to this specialised trauma have been categorised and discussed in some detail particularly in their later stages. The enhanced vulnerability of the irradiated microvasculature in diabetic patients ${ }^{10,27}$ and those receiving concomitant chemotherapy has also been remarked upon in the literature. $^{28}$

The earliest post-radiation microvascular changes in the retina have received much less attention, partly because they are asymptomatic and difficult to detect in the clinical situation. Furthermore, the variable and unpredictable latent period which predates the development of observable retinopathy compounds the difficulty of identifying early disease particularly in patients who may be severely ill but with no ocular complaints. The pathogeneses of the initial microvascular events leading to capillary incompetence, closure and proliferation are also far from clear, and although it has been suggested that 


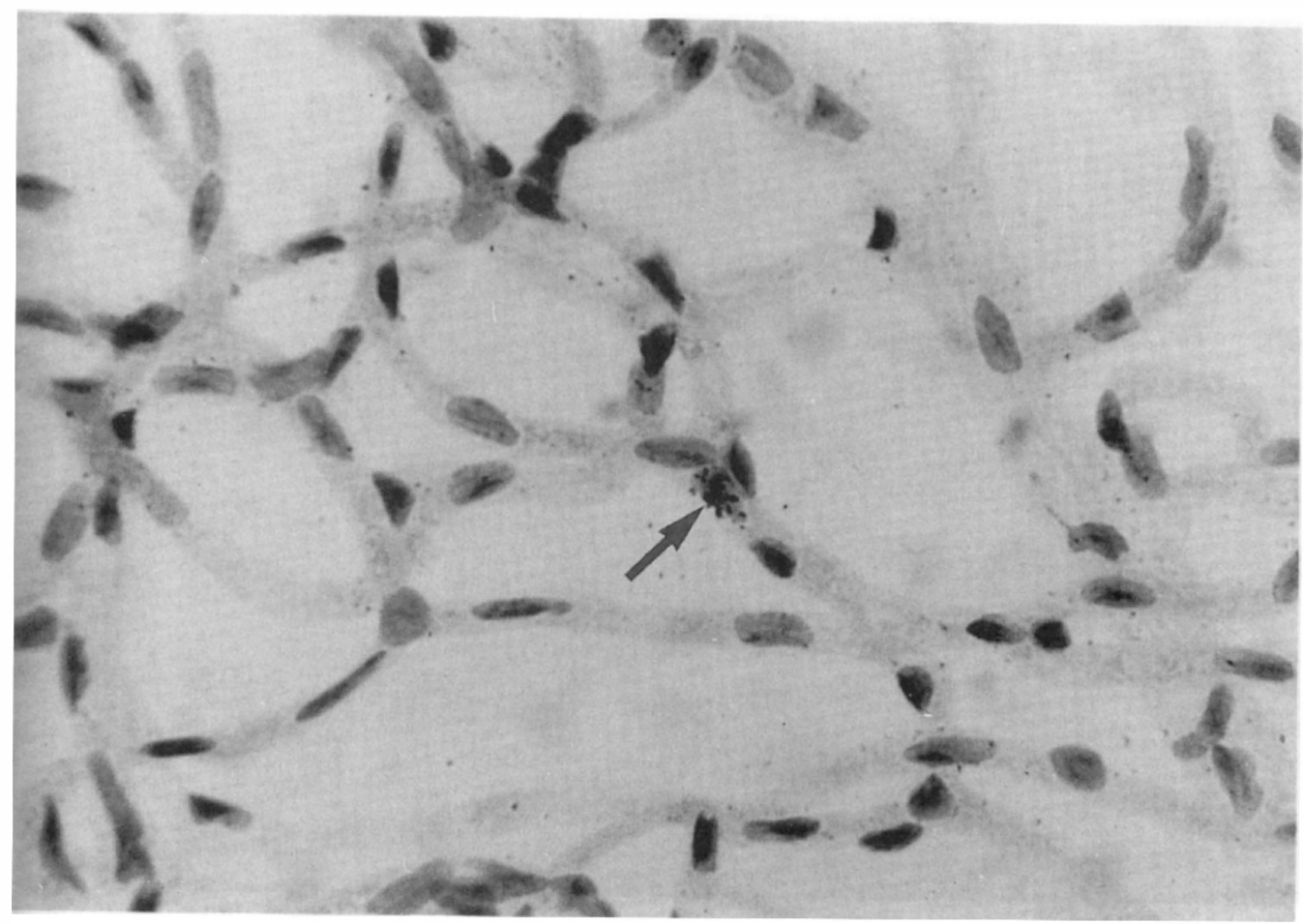

Fig. 10. Trypsin digest autoradiogram of rat retinal vasculature following 48-hour infusion of $3 H$-thymidine. A labelled endothelial cell nucleus is heavily decorated with silver grains (arrow). Orig. Mag. $\times 500$

the vascular endothelium is the key cell in the initiation of the disease process this has not to this time been convincingly demonstrated. ${ }^{8}$

The clinico-pathological and experimental investigations reported in this paper were undertaken to identify and elucidate the earliest retinal microvascular changes induced by radiation and detect those alterations in the vascular cells which precipitate the vascular occlusion and incompetence characteristic of radiation retinopathy.

Clinical and pathological studies of early radiation retinopathy and experimental investigations in the rat demonstrate the particular susceptibility of vascular endothelial cells to ionising radiation. Gardiner and Amoaku in our laboratory have shown that the highly heterochromatic nuclei of rat photoreceptor cells are exquisitely sensitive to ionising radiation compared to those of the primate which are more euchromatic. ${ }^{20,29}$ Similarly the inner retinal neurones, particularly the ganglion cells, are highly euchromatic and have been shown to be very radioresistant. It would therefore appear that tightly packed heterochromatin is more vulnerable to radiation damage than finely dispersed euchromatin and may in part explain the radiosensitivity of the vascular endothelial cells, the nuclei of which are relatively heterochromatic. The hypersensitivity of cells undergoing mitosis further illustrates the severe impact of radiation on tightly packed nuclear chromatin which in mitotic cells is maximally condensed as chromosomal bodies.

Environmental factors also probably contribute to the radiosensitivity of vascular endothelium, particularly the high oxygen tension on the arterial side of the circulation and the direct exposure of the endothelial cell to some blood constituents, e.g. iron conjugates or iron-containing proteins, which may promote or accentuate free radical damage. The insulation of the pericyte from the blood stream by the endothelium may account for its relative resistance to radiation and its persistance in many irradiated capillaries despite the demise of endothelial cells. 


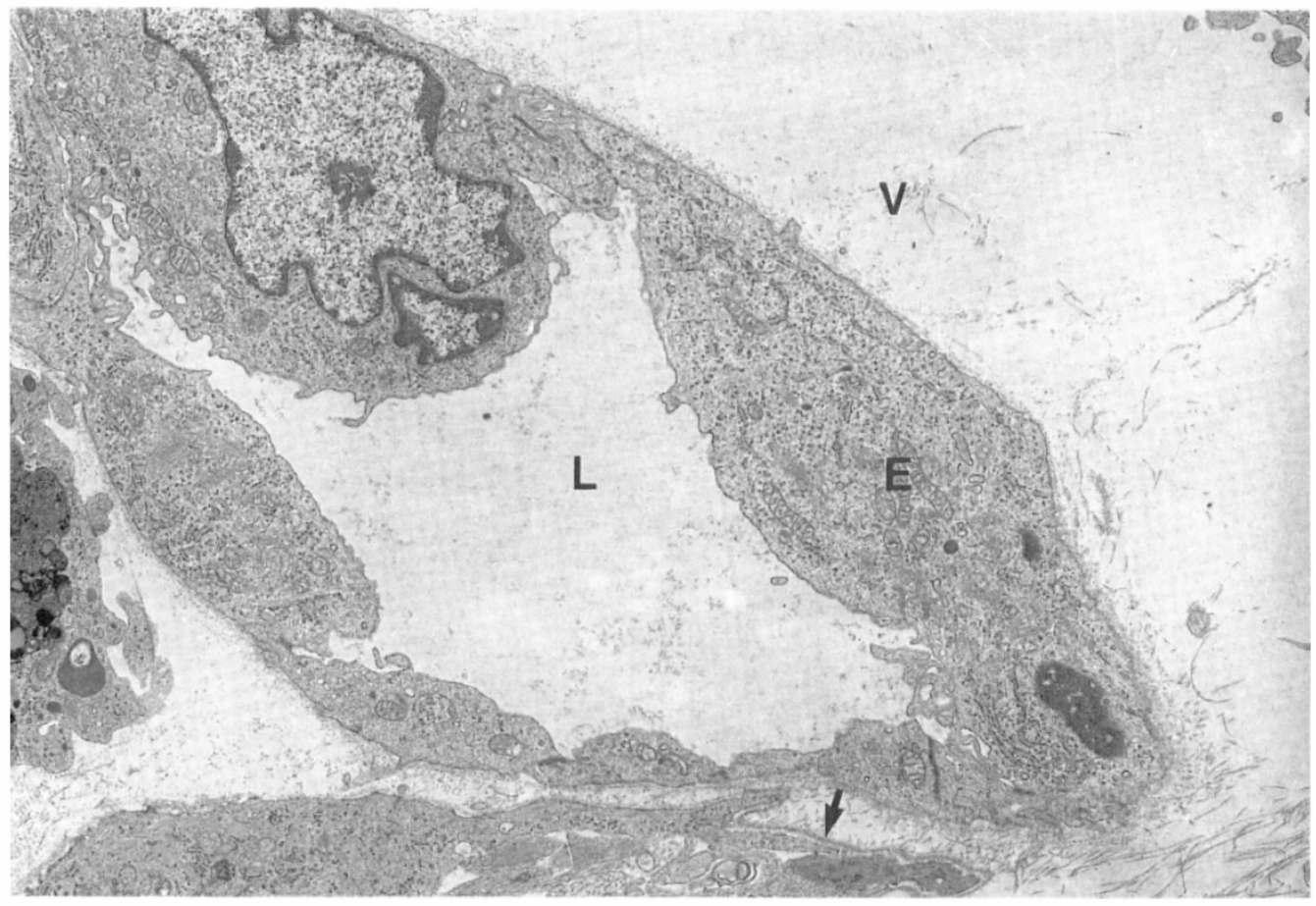

Fig. 11. Electron micrograph of $S T Z$ diabetic rat one year after 1,500 cGy of $x$-radiation shows a new retinal vessel (lumen $L$ ) just beyond its point of entry into the vitreous body $(V)$. The vessel consists of plump hypertrophic endothelial cells $(E)$ surrounded by a thin flocculent basal lamina. Internal limiting membrane of the retina (arrow). Orig. Mag. $\times 9000$

The precise cellular events leading to capillary occlusion are not fully established however, the above investigations would suggest that a scattered population of endothelial cells die at or soon after irradiation. Such cells may suffer immediate mitotic death or interphase death and the higher the radiation dose the greater the number of cells which incur such damage. This first wave of cell death also compels adjacent cells to divide to repair the initial endothelial defects. Some of these neighbouring cells will have sustained radiation damage and the forced division may precipitate further mitotic death. This sequence of events may be repeated many times until the surviving healthy endothelial cells, even though stretched and elongated, are insufficient to maintain a continuous endothelium and the clotting cascade is activated with subsequent capillary occlusion.

With time, fresh waves of vascular endothelial cell loss can be anticipated as irreparably damaged cells undergo mitotic death whilst attempting to divide in the normal cycle of endothelial cell renewal thereby initiating a further round of local cell death and vascular occlusion. The accelerated rate of division of retinal vascular endothelial cells noted in the diabetic Wistar rat ${ }^{30}$ may explain in part the known susceptibility of the retinal vessels in diabetic patients to radiation. New foci of endothelial cell loss may also result from the delayed interphase death of cells that have exhausted vital cytoplasmic components which cannot be replaced because of nuclear damage.

As affected capillaries become occluded, blood is diverted into nearby channels which may already be compromised by endothelial cell damage or pericyte loss. These vessels dilate under the haemodynamic load and become clinically apparent as capillary collaterals which may or may not maintain competence. Structural deficiencies in the vessel wall coupled with increased blood flow produce capillary irregularities, fusiform outpouchings of the basement membrane and saccular microaneurysms which seem to pre- 


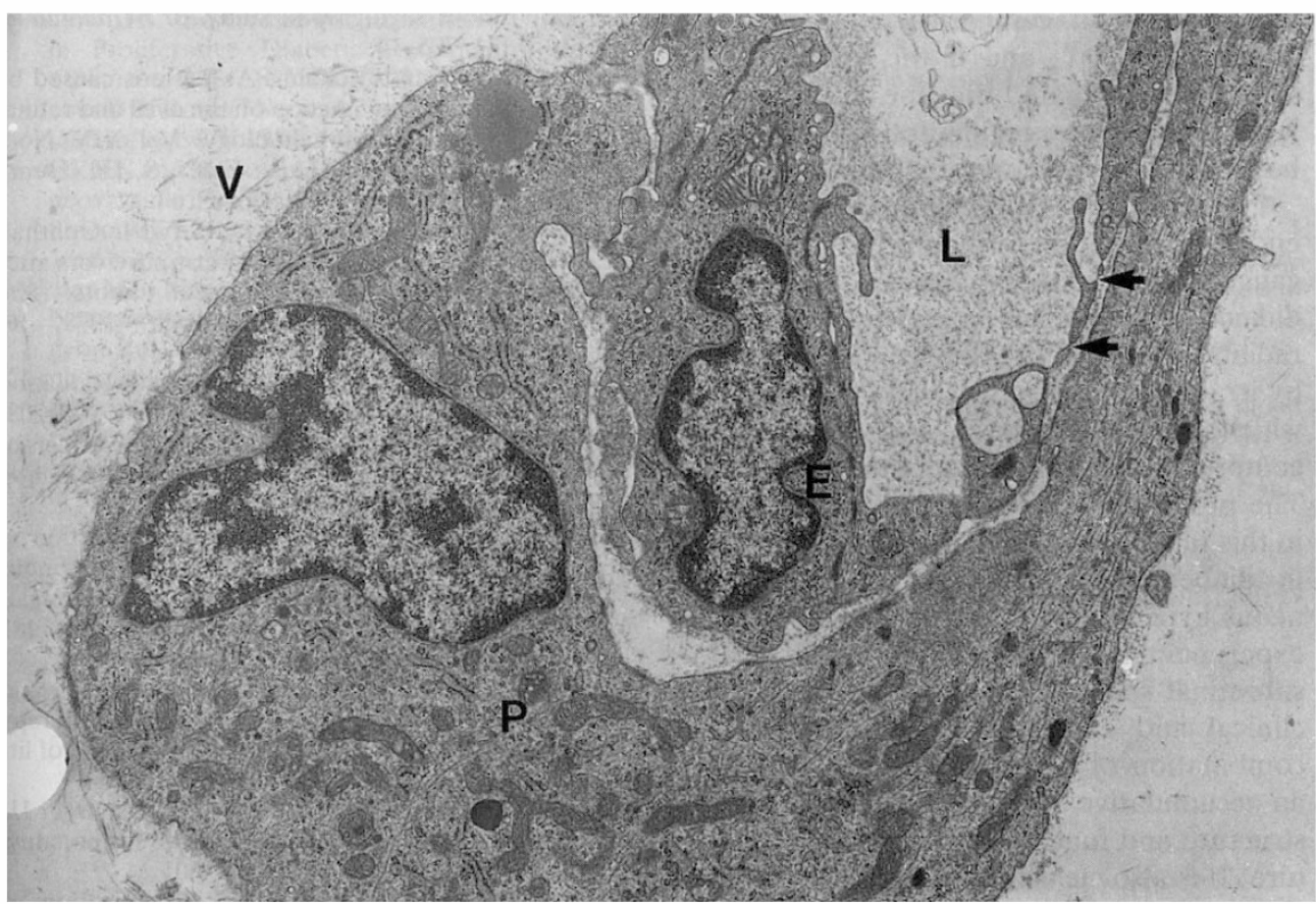

Fig. 12. Electron micrograph of STZ diabetic rat one year after 1,500 cGy of x-radiation shows a new retinovitreal vessel (lumen L) close to the retinal surface. The endothelial cell (E) shows several fenestrations (arrows) and is surrounded by a large hypertrophic pericyte $(P)$ rich in cell organelles. Vitreous (V). Orig. Mag. $\times 6500$

dominate on the arterial side of the circulation and at the junction of perfused and non-perfused channels. The microaneurysms vary in size and shape and typically display a cycle of expansion, leakage, thrombosis and occlusion.

Many of the capillary collaterals and associated venules attain sizeable dimensions under the haemodynamic load, become incompetent and assume a telangiectatic-like appearance which is typical if not pathognomonic of radiation retinopathy. These dilated channels become encapsulated within a collagen-rich adventitia which separates them from the neighbouring oedematous, necrotic and gliotic retinal parenchyma. The frequent presence of fenestrated endothelial cells in these telangiectatic-like channels not only accounts for their striking incompetence, but also suggests that the degeneration of the normal retinal neuropile is conducive to the elaboration of a less specialised endothelium, as occurs in most capillaries outside the eye and central nervous system.
The loss of capillary network is insidious and progressive; however, the rate of change is typically slow and if macular oedema does not extend to the foveola visual functions remain good. There is little in the way of capillary regeneration or recanalisation although defunct larger channels, particularly arterioles, may be invaded by new capillaries which may have fenestrated endothelial cells. Where radiation damage is intense, precapillary arteriolar occlusion with widespread inner retinal ischaemia may complicate the above picture, and the intraretinal accumulation of blood, plasma constituents and neuronal debris corresponds to the degree of ischaemia and microvascular competence.

The macular microvasculature appears to be more vulnerable to radiation trauma subsequent to teletherapy involving the retina. The reason for this is not clear; however, it may be a function of capillary density and high oxygen levels in this part of the retina with an inordinately high unit blood flow. It has also been noted that endothelial cell turnover is 
higher in small capillaries compared with larger channels ${ }^{31}$, and if this is the case it would be anticipated that the macular capillaries with putative high mitotic indices would be more susceptible to radiation.

Preretinal neovascularisation is a feature of end-stage radiation retinopathy and a further gauge of the extent of ischaemia. Such vessels did not develop in our animal model of simple radiation retinopathy, but could be induced by irradiating the eyes of diabetic animals in which the retinal vasculature was already compromised by several months of diabetes. The preretinal new vessels which developed in this model were similar to those described in diabetes, ${ }^{21,22}$ retinal tumours, ${ }^{23}$ spontaneous hypertensive ${ }^{24}$ and RCS rats, ${ }^{25}$ and in experimental new choroidal vessels in the subretinal space. ${ }^{26}$ It is apparent both from clinical and experimental studies that the combination of diabetes and radiation have an accumulative and damaging effect on the structure and function of the retinal vasculature. It is also clear that the pathological picture is one of an ischaemic retinopathy and that neovascularisation which may be a sequel of either condition is a direct result of retinal ischaemia.

Most patients receiving radiation doses of less than $2500 \mathrm{cGy}$ in fractions of $200 \mathrm{cGy}$ or less are unlikely to develop significant retinopathy. Patients with mild retinopathy typically progress only very slowly. and visual functions remain good unless there is significant macular involvement. A minority of patients develop sight threatening macular oedema and may respond to focal laser photocoagulation which can reduce the extent of intraretinal oedema and improve the level of competence in some affected capillaries. ${ }^{19}$ Occasionally patients subjected to large doses of radiation may develop preretinal and papillary neovascularisation and benefit from pan retinal photocoagulation or vitreo-retinal surgery in selected cases. ${ }^{14}$

\footnotetext{
References

${ }^{1}$ Moore RF: The value of radium in intraocular lesions. Trans Ophthalmol Soc UK 1935, 55: 3-26.

${ }^{2}$ Stallard HB: Glioma retinae treated by radon seeds. Br Med J 1936, 11: 962-4.

${ }^{3}$ Hayreh SS: Post-radiation retinopathy: A fluores-
}

cein fundus angiographic study. Br J Ophthalmol 1970, 74: 705-14.

${ }^{4}$ Duke-Elder S and McFaul PA: Lesions caused by ionising radiation: Action on the uvea and retina. In System of Ophthalmology. Vol XIV Nonmechanical Injuries. Duke-Elder, S, Ed. Henry Kimpton London. 1972, 976-82.

${ }^{5}$ de Schryver A, Wachmaster L, Baryd I: Ophthalmology observations on long-term survivors after radiotherapy for nasopharyngeal tumors. Acta Radiol Ther Phys Biol (Stockh) 1972, 10: 193-209.

${ }^{6}$ Amoaku WMK and Archer DB: Cephalic Radiation and Retinal Vasculopathy. Eye 1990, 4: 195-203.

${ }^{7}$ Merriam GRJ, Szechter A, Focht EF: The effects of ionising radiation on the eye. Front Radiation Ther ONC 1972, 6: 346-85.

${ }^{8}$ Ehlers $\mathrm{N}$ and Kaae S: Effects of ionising radiation on retinoblastoma and on the normal ocular fundus in infants. A photographic and fluorescein angiographic study. Acta Ophthalmol (Suppl) 181 (Copenh) 1987, 65: 3-84.

${ }^{9}$ Nakissa N, Rubin P, Strohl R, Keys H: Ocular and orbital complications following radiation therapy of paranasal sinus malignancies and review of literature. Cancer 1983, 51: 980-6.

${ }^{10}$ Brown GC, Shields JA, Sanborn G, Augsburger JJ, Savino PJ, Schatz NJ: Radiation retinopathy. Ophthalmology 1982, 89: 1494-501.

${ }^{11}$ Char DH, Lonn LI, Margolis LW: Complications of cobalt plaque therapy of choroidal melanomas. Am J Ophthalmol 1977, 84: 536-41.

${ }^{12}$ Wara WM, Irvine AR, Neger RE, Howes EL, Phillips TL: Radiation retinopathy. Int J Radiat Oncol Biol Phys 1979, 5: 81-3.

${ }^{13}$ Midena E, Segato T, Piermarocchi S, Corti L, Zorat PL, Moro F: Retinopathy following radiation therapy of paranasal sinus and nasopharyngeal carcinoma. Retina 1987, 7: 141-7.

${ }^{14}$ Kinyoun JL, Kalina RE, Brower SA, Mills RP, Johnson RH: Radiation retinopathy after orbital irradiation for Graves' ophthalmopathy. Arch Ophthalmol 1984, 102: 1473-6.

${ }^{15}$ Gass JDM: A fluorescein angiographic study of macular dysfunction secondary to retinal vascular disease. Arch Ophthalmol 1968, 80: 606-17.

${ }^{16}$ Chee PHY: Radiation retinopathy. Am J Ophthalmol 1968, 66: 860-5.

${ }^{17}$ Chaudhuri PR, Austin DJ, Rosenthal AR: Treatment of radiation retinopathy. $\mathrm{Br} \mathrm{J}$ Ophthalmol 1981, 65: 623-5.

${ }^{18}$ Parsons JT, Fitzgerald CR, Hood I, Ellingwood KE, Bova FJ, Million RR: The effects of irradiation on the eye and optic nerve. Int J Radiat Oncol Biol Phys 1983, 9: 609-22.

${ }^{19}$ Amoaku WMK and Archer DB: Fluorescein angiographic features, natural course and treatment of radiation retinopathy. Eye 1990, 4 (in press).

${ }^{20}$ Amoaku WMK: Thesis. The effects of ionizing radiation on the retina: A clinical and experimental study. Queen's University Belfast, June 1990.

${ }^{21}$ Taniguchi Y: Ultrastructure of newly formed blood vessels in diabetic retinopathy. Jap J Ophthalmol 1976, 20: 19-31. 
${ }^{22}$ Wallow IHL and Gedler PS: Endothelial Fenestrae in Proliferative Diabetic Retinopathy. Invest Ophthalmol Vis Sci 1980, 19: 1176-83.

${ }^{23}$ Archer DB: Retinal Neovascularization. Trans Ophthalmol Soc UK 1983, 103: 2-27.

${ }^{24}$ Frank RN and Mancini MA: Presumed retinovitreal neovascularization in dystrophic retinas of spontaneously hypertensive rats. Invest Ophthalmol Vis Sci 1986, 27: 346-55.

${ }^{25}$ Weber ML, Mancini MA, Frank RN: Retinovitreal Neovascularization in the Royal College of Surgeon Rat. Curr Eye Res 1989, 8: 61-74.

${ }^{26}$ Archer DB and Gardiner TA: Morphologic, Fluorescein angiographic and light microscopic features of experimental choroidal neovascularization. Am J Ophthalmol 1981, 91: 297-311.

${ }^{27}$ Dhir SP, Joshi AV, Banerjee AK: Radiation ret- inopathy in diabetes mellitus. Acta Radial Oncol 1982, 21: 111-3.

${ }^{28}$ Chan RC and Shukovsky LJ: Effects of irradiation on the eye. Radiology 1976, 120: 673-5.

${ }^{29}$ Amoaku WMK, Frew L, Mahon GJ, Gardiner TA, Archer DB: Early ultrastructural changes after low-dose $\mathrm{x}$-irradiation in the retina of the rat. Eye 1989, 3: 638-46.

${ }^{30}$ Sharma NK, Archer DB, Gardiner TA: A morphologic and autoradiographic study of cell death and regeneration in the retinal microvasculature of normal and diabetic rats. Am J Ophthalmol 1985, 100: $51-60$.

${ }^{31}$ Reinhold HS: The influence of radiation on blood vessels and circulation. Chapter 2 Cell viability of the vessel wall. In Current Topics in Radiation Research Quarterly. North-Holland 1974: 9-28. 